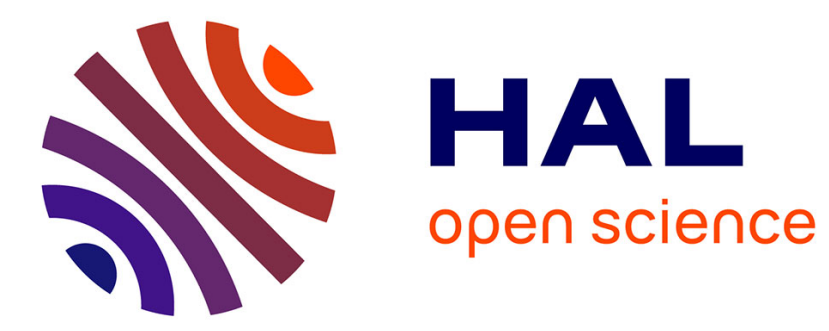

\title{
Development of models for additively manufactured actuators using compliant Wren mechanism
}

Lennart Rubbert, Felix Schuler, Thibault Gayral, Michael de Wild, Pierre

Renaud

\section{- To cite this version:}

Lennart Rubbert, Felix Schuler, Thibault Gayral, Michael de Wild, Pierre Renaud. Development of models for additively manufactured actuators using compliant Wren mechanism. Precision Engineering, 2021, 72, 10.1016/j.precisioneng.2021.05.002 . hal-03257414

\section{HAL Id: hal-03257414 \\ https://hal.science/hal-03257414}

Submitted on 10 Jun 2021

HAL is a multi-disciplinary open access archive for the deposit and dissemination of scientific research documents, whether they are published or not. The documents may come from teaching and research institutions in France or abroad, or from public or private research centers.
L'archive ouverte pluridisciplinaire HAL, est destinée au dépôt et à la diffusion de documents scientifiques de niveau recherche, publiés ou non, émanant des établissements d'enseignement et de recherche français ou étrangers, des laboratoires publics ou privés. 


\title{
Development of models for additively manufactured actuators using compliant Wren mechanism
}

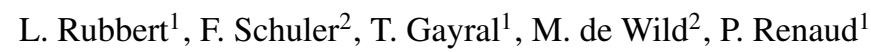 \\ ${ }^{1}$ ICube, CNRS - University of Strasbourg - INSA Strasbourg, France \\ ${ }^{2}$ University of Applied Sciences Northwestern Switzerland, FHNW, Switzerland.
}

\begin{abstract}
Compliant Wren mechanisms (CWM) constitute specific compliant structures of particular interest. Derived from Wren mechanisms, they can exhibit a large variety of motions, from quasi translation to quasi rotation. In this paper, the development of models for the analysis and synthesis of CWM is considered. A kinematic model is introduced first to assess all possible motions when used as an actuator. Then the static model and stress expressions are derived to help their design. These derivations are achieved for two types of geometries, corresponding to the geometries of interest. CWM are filigree structures, whose manufacturing is difficult to consider without additive manufacturing. A specific work on their production using selective laser melting (SLM) is then achieved to ensure the reliability of their production. As a proof of concept, a pneumatically actuated component is then developed and tested. It is composed of two CWM of different geometries. It offers the possibility to obtain translation and rotation using a single pressure input. The developed models are investigated using finite element models and experiments using additively manufactured structures.
\end{abstract}

Keywords: Wren mechanism, compliant mechanism, additive manufacturing, titanium

\section{Introduction}

The interest of parallel mechanisms is well identified and exploited in robotic manipulation [1]. In addition, specific motions can be generated with, for instance, helical motion using 3-US mechanism [2, 3] or Wren mechanism [4]. One has then the advantage of a parallel structure in terms of stiffness, and at the same time the possibility to adjust the mechanism kinematics for given requirements [5]. The Wren mechanism was studied for deployable and reconfigurable mechanisms [6, 7] and it is of particular interest. The kinematic properties of the Wren mechanism have been analyzed in details in [8, 9]. When the mechanism legs are not parallel, the mechanism presents three infinitesimal motions and it has one finite mobility, which is an helical motion with non-con- stant pitch of its platform. The pitch of this helical motion can be adjusted between 0 and infinity depending on the direction of the legs. We are thus interested in this article by the finite motion of this mechanism, used as a generator of screw motion of variable pitch.

In [4], the Wren mechanism was being used as a structure for a rotary actuator, in combination with pneumatic actuation. The Wren mechanism was then designed and produced as a compliant structure in order to benefit from the accuracy of compliant mechanisms in combination with fluidic actuation. The implementation can be conducted either for small stroke by simply using a chamber reinforced by a compliant Wren mechanism (CWM), or by integrating the device in a step by step system [10, 11]. In biomedical context, design of compliant actuators for needle manipulation using such 


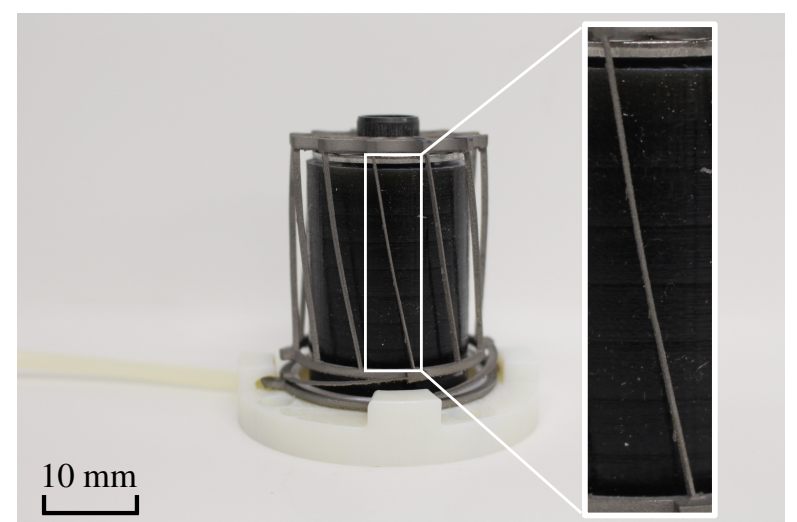

Figure 1: Prototype of pneumatic actuator for biomedical application based on two 3D printed compliant Wren mechanisms.

an approach is of interest [12,13]. This is our application context in the paper (Fig. 1).

Two aspects need to be investigated in order to build actuators with CWM. First, there is no model for the design of CWM. In [5] a compliant linear-rotation motion transduction element is presented. In [14] a concept for dissipating kinetic energy using arrays of CWM is introduced. In [14], the FACT design method is applied to design a non-conventional CWM. But to our knowledge there is no model to guide the geometrical synthesis of a CWM for given desired kinematics: the link between the finite motion properties and the CWM geometry has to be established. Second, a CWM is a filigree structure, with a complex 3D geometry as it can be seen in figure 1. If produced with conventional processes, the need of assembling several components will affect the compactness, and also the stiffness which may impact the specific kinematics of the structure. Additive manufacturing (AM) is a natural choice given the freedom of design. Selective Laser Melting (SLM) is well established for the manufacturing of reliable titanium structures, that exhibit large elastic domain. SLM is indeed a very potent technology for advanced manufacturing but it also has inherent disadvantages: the layer-by-layer process is challenging for fine, free-standing structures with long aspect ratio and for structural transitions from thin to bulky geometries [15]. Filigree structures suffer especially from inherent process limitations like the particle size of the used powder, the size and shape of the laser melting zone and the inhomogeneity in the mechanical distribution of the new powder layer [16].

The paper therefore contains 2 contributions to develop the concept of actuators based on CWM for precision engineering. The first one is the introduction of models for the design, and the second one is AM strategy for their manufacturing. In order to develop both aspects, a proof of concept of actuator for biomedical applications is considered. In section 2, the principle of the developed component is introduced. Then in section 3, models are derived to determine geometries of interest for a given set of requirements. Two models are elaborated to cover the large possible range of kinematics. In section 4, the determination of AM strategy based on experimental approach is presented together with the associated design for additive manufacturing guidelines. The proof of concept of actuator for medical applications is designed and tested in section 5, before concluding in section 6 .

\section{Principle of the developed component}

\subsection{Clinical requirements and intended use}

The component we develop is dedicated to imageguided procedures. In interventional radiology, physicians perform needle insertion in a patient body to sample tissues for diagnostic [17]. When X-ray based imaging device is used to guide the insertion, the presence of ionising radiations represents a safety concern for the physician. It is then desired to control remotely the needle motion. Needle translation is needed, as well as the needle self-rotation, as the needle bevel asymmetry can be used to adjust the needle path [18]. For needle steering purpose, our goal is here to have a device that can be used to translate and rotate the needle. Space in the imaging device is very limited. Step by step motion as performed in [13] is therefore selected.

Compressed air is available in the operating room. We then desire to have a pneumatic chamber to develop forces for the needle translation and rotation. Following [13], the operating pressure is set to 3 bars, and intended displacement and rotation are equal to $2 \mathrm{~mm}$ and approximately $5^{\circ}$. In soft tissues such as liver or kidney, the needle is in interaction with tissues of very low stiffness and the insertion forces are low [19], so they are neglected during analysis.

\subsection{Principle}

Pneumatic actuation is interesting to ensure compatibility with the operating room constraints. Having only one pneumatic chamber to control the two desired motions would be in favor of the component compactness. A design based on a single pneumatic chamber is then proposed. The component we consider is built upon a compliant structure which is composed of 2 CWM, designated as modules 1 and 2 on figure 2. The 2 CWM 


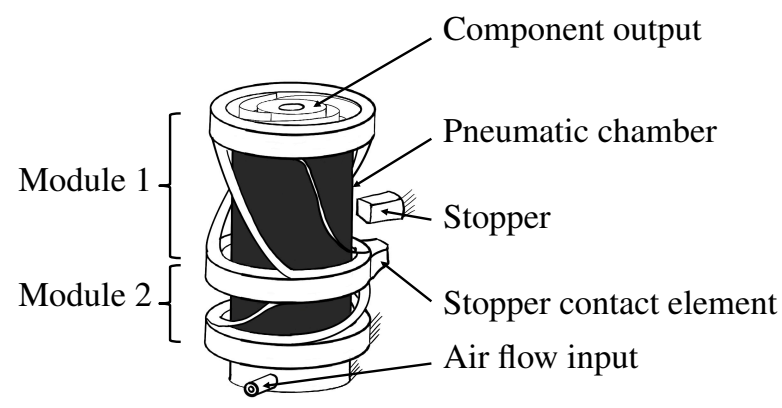

Figure 2: Schematic view of the developed component

have opposite pitches, so they have both helical motions but of opposite sense. The pneumatic chamber generates an axial force on the compliant structure to induce the motion of the component output.

A mechanical stopper is used to control the output motion, as schematically represented in figure 3 When the input pressure is below a threshold $p_{t}$, the output motion is a translation, thanks to the combined motions of the two CWM of opposite pitches. When the pressure reaches the threshold, the upper part of the module 1 is axially constrained by the stopper and the rotation is compensated. Then, the increase of pressure only causes the deformation of the module 2 , as represented in the figure 3, so a rotational motion is obtained.

\section{Modeling of compliant Wren mechanism}

\subsection{Parameterization}

A CWM is composed of $n$ identical helical beams connecting the base to the platform (Fig. 4). Their arrangement is axisymmetric. The mechanism height is denoted $H$. The arrangement of flexible beams is set by the radius $R$ defined as the distance between the mechanism axis of revolution and the neutral fibers of the beams. The helical shape is set by the pitch $p$, such that the angle $\gamma=h / p$ (Fig. 4) and the beams have a rectangular section with a thickness $t$ in the tangential direction and width $w$ in the radial direction as represented in figure 4

The set of parameters $(n, H, R, p, t, w)$ defines the CWM geometry, with the neutral fiber of each flexible beam defined by

$$
x=R \cos \left(\frac{z}{p}\right), y=R \sin \left(\frac{z}{p}\right), z \in[0 ; H]
$$

The length $L$ of the beam neutral fiber can then com-
Module 1

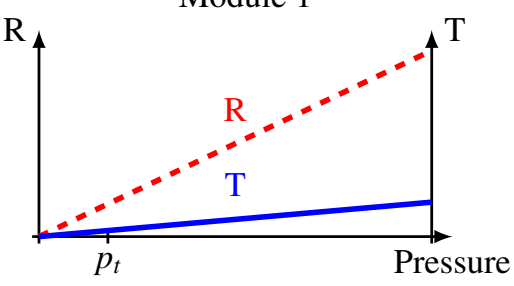

Module 2

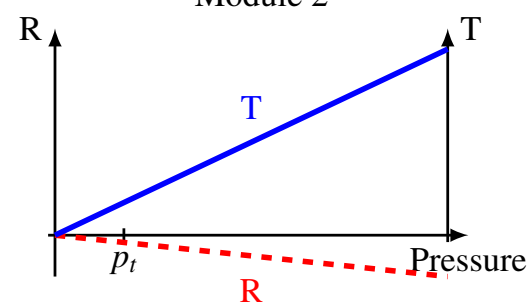

Overall motion

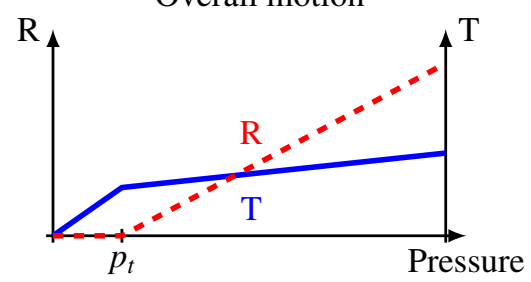

Figure 3: Rotation $(\mathrm{R})$ and translation $(\mathrm{T})$ of the two modules and the desired overall actuator motion

puted by

$$
L=\int_{0}^{H} \sqrt{\left(\frac{R}{p} \times d z\right)^{2}+d z^{2}}=\sqrt{\left(\frac{R}{p}\right)^{2}+1} \times H
$$

It is in addition interesting to define the inclination angle $\alpha$ of the helical neutral fiber with the vertical axis since this latter is the projection of a straight line on a cylinder:

$$
\alpha=\arccos \left(\frac{H}{L}\right)
$$

\subsection{Kinematic model}

For the design of a CWM, it is needed to establish first the relationship between the axial displacement $u$ (Fig. 4) and the rotation $\theta$ of the platform. The platform motion is made possible by the deformation of the flexible beams under the application of an axial force $N$ on the structure. To be of practical interest, the CWM must offer a significant lateral stiffness to have only one degree of freedom. The flexible beams must then possess a small section compared to the platform radius: $(w, t) \ll R$. The beam width is much larger than the 


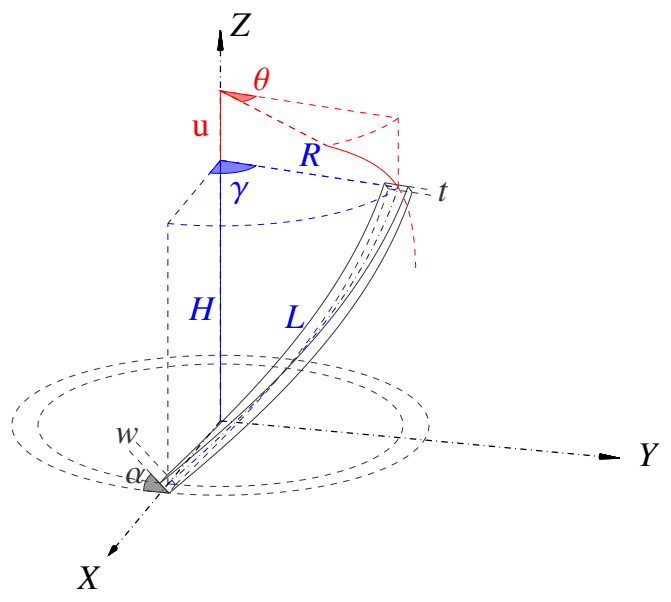

Figure 4: CWM parameterization. Only one beam is represented for sake of clarity.

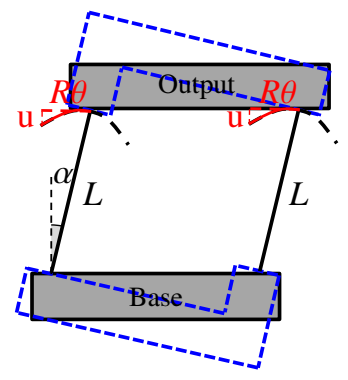

(a) Unwrapped Wren mechanism composed of two beams.

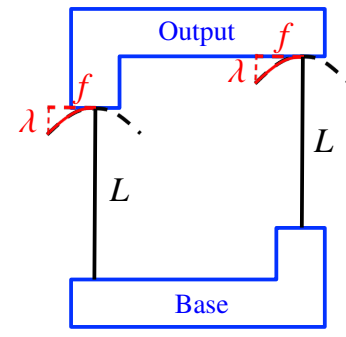

(b) Parallel-spring stage as obtained after rotation of the structure.
Figure 5: Similarity of Wren structure after unwrapping and parallelspring stage.

beam thickness $(w \gg t)$ so the bending of beams provides the helical motion of the platform. The beams also have a length $L$ which is much greater than the thickness $t$ and width $w: L \gg(w, t)$

In these conditions, it is possible to analyse the CWM by virtually unwrapping the structure to make it planar, and then analyse its kinematics. The structure is then an assembly of flexible beams, which behaviour can be inferred from the analysis of a parallel spring stage as performed in [20]. The structure after unfolding is represented in a simplified situation with two beams in figure 5 (a). The motions of the output elements in figure 5(a) and figure 5(b) are similar. Thus we can deduce the non-linear CWM kinematic relationship between the axial displacement $u$ and the axial rotation $\theta$ from the tangential motion $f$ of the parallel spring stage and its parasitic motion $\lambda$. These motions were analysed in [20].

In fact, for a parallel spring stage, the motion exhib- ited by the output element is parabolic. Its tangential displacement $f$ is related to the displacement $\lambda$ in the beam direction with

$$
\lambda=-\frac{3 f^{2}}{5 L} .
$$

This means the projection the displacement $R \theta$ in the base frame (Fig. 5(a)) is

$$
R \theta=f \cos (\alpha)-\lambda \sin (\alpha) .
$$

So the output rotation $\theta$ is

$$
\theta=\frac{f \cos (\alpha)-\lambda \sin (\alpha)}{R}
$$

Using again figure 5(a), the axial displacement $u$ is

$$
u=\lambda \cos (\alpha)+f \sin (\alpha)
$$

The displacement $f$ can therefore be determined by combining (6) and (4):

$$
f \cos (\alpha)+\frac{3 f^{2}}{5 L} \sin (\alpha)-\theta R=0
$$

which gives two solutions

$f_{1,2}=-\frac{5 L}{6 \sin (\alpha)}\left(\cos (\alpha) \pm \sqrt{\frac{5 L \cos (\alpha)^{2}+12 R \theta \sin (\alpha)}{5 L}}\right)$

Thus $u_{1}$ and $u_{2}$ are obtained by combining equations (9) with equation (7).

$$
\begin{array}{r}
u_{1,2}=-\frac{5 L \cos (\alpha)+6 R \theta \cos (\alpha) \sin (\alpha)}{6 \sin (\alpha)^{2}} \\
\pm \frac{\sqrt{5} L \sqrt{\frac{5 L \cos (\alpha)^{2}+12 R \theta \sin (\alpha)}{L}}}{6 \sin (\alpha)^{2}}
\end{array}
$$

For both solutions, the relationship between translation and rotation can be represented by a set of curves like the ones represented in figure 6. We can observe the variety of motions that can be obtained with the CWM. Two main regions are of particular interest. For small inclination angle $\alpha$ of beams, the output motion is a quasi translation. For large inclination angle $\alpha$, quasi rotation can be observed. Both behaviors are of interest. For each of the two corresponding types of CWM, we thus derive the force-displacement model, and the maximum stress in the beams for the CWM design. 


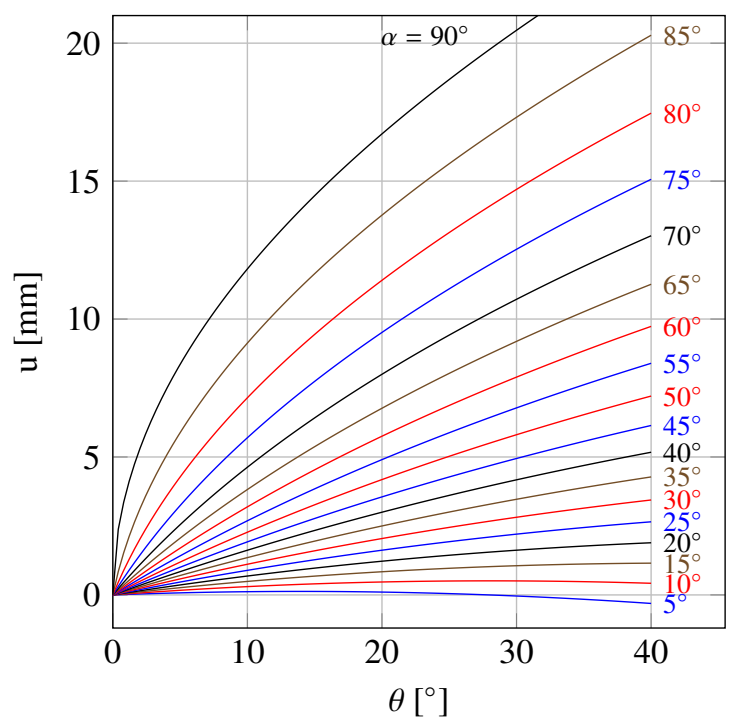

Figure 6: Representation of possible relationships between the axial displacement $u$ and the rotation $\theta$ for different inclination angle $\alpha$. The curves are plotted for $L=40 \mathrm{~mm}$ and $R=12 \mathrm{~mm}$, corresponding to the prototype of figure 1 .

\subsection{CWM with small inclination angle}

\subsubsection{Stiffness determination}

For small values of the angle $\alpha$, the CWM is composed of $n$ curved beams that lie in a quasi-planar configuration (Fig. 7). The application of the vertical load $N$ then mostly creates the bending of the beams. For small inclination angles, we therefore assume the model linking the axial load to the platform motion can be derived by only considering the bending of the curved beams.

A single beam is submitted to an axial load $N / n$. The

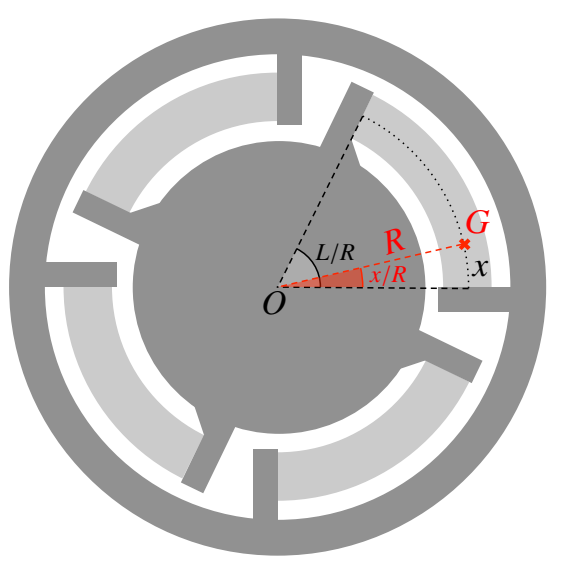

Figure 7: Parameterization for CWM with small inclination angle. bending moment $M_{f}$ then evolves along the neutral fiber with

$$
M_{f}=\frac{N}{n} R \sin \left(\frac{\frac{L}{2}-x}{R}\right) .
$$

The vertical displacement along the beam axis $u$ is then obtained by integration of the relationship

$$
u^{\prime \prime}=\frac{M_{f}}{E I}
$$

with $E$ the Young's modulus of the CWM material and I the area moment of inertia of the beam. One has then

$u^{\prime}=\frac{N R}{n E I} \int_{0}^{x} \sin \left(\frac{\frac{L}{2}-x}{R}\right) d x+A=\frac{N R^{2}}{n E I} \cos \left(\frac{\frac{L}{2}-x}{R}\right)+A$

From the boundary condition $y^{\prime}(L)=0$,

$$
A=-\frac{N R^{2} \cos \left(\frac{L}{2 R}\right)}{n E I}
$$

so the vertical displacement is

$$
\begin{aligned}
& u=\frac{N R^{2}}{n E I} \int_{0}^{x} \cos \left(\frac{\frac{L}{2}-x}{R}\right)-\cos \left(\frac{L}{2 R}\right) d x+B \\
& u=-\frac{N R^{2}}{n E I}\left(R \sin \left(\frac{\frac{L}{2}-x}{R}\right)+\cos \left(\frac{L}{2 R}\right) x\right)+B
\end{aligned}
$$

With the boundary condition $y(0)=0$ :

$$
B=\frac{N R^{2}}{n E I}\left(R \sin \left(\frac{L}{2 R}\right)\right)
$$

and finally

$$
u=-\frac{N R^{2}}{n E I}\left(R \sin \left(\frac{\frac{L}{2}-x}{R}\right)+\cos \left(\frac{L}{2 R}\right) x-R \sin \left(\frac{L}{2 R}\right)\right)
$$

The vertical displacement of the CWM under the application of the axial load $N$ is then equal to

$$
u(L)=\frac{N R^{2}}{n E I}\left(2 R \sin \left(\frac{L}{2 R}\right)-L \cos \left(\frac{L}{2 R}\right)\right)
$$

which becomes when introducing the value of the area moment of inertia $I=\frac{w t^{3}}{12}$ :

$$
u(L)=\frac{24 N R^{3}}{n E w t^{3}}\left(\sin \left(\frac{L}{2 R}\right)-\frac{L}{2 R} \cos \left(\frac{L}{2 R}\right)\right)
$$

The CWM axial stiffness $K_{c}$ can therefore be expressed as the ratio between the axial load $N$ and the vertical displacement $u$ :

$$
K_{c}=n \frac{E w t^{3}}{24 R^{3}} \frac{1}{\left(\sin \left(\frac{L}{2 R}\right)-\frac{L}{2 R} \cos \left(\frac{L}{2 R}\right)\right)}
$$




\subsubsection{Stress estimation}

The stress in the CWM beams is expressed from the value of bending stress for a beam under the deflection $f$ :

$$
\sigma_{b}=3|f| \frac{E t}{L^{2}} .
$$

\section{4. $C W M$ with large inclination angle}

\subsubsection{Stiffness determination}

When submitted to the axial load $N$, the CWM platform exhibits an helical motion. The flexible beams are then being submitted to bending, traction and torsion. The CWM behavior is established by considering each of the three solicitations separately, and then computing the force-displacement relationship.

The bending stiffness of a parallel spring stage can be approximated by [20]:

$$
K_{b}=\frac{24 E I}{L^{3}}+\frac{6 F_{A}}{5 L},
$$

with $I=\frac{w t^{3}}{12}$ and $F_{A}$ is the load in the direction of the blades (Fig. 8). Here, the CWM is composed of $n$ beams, so each of the is being submitted to a tangential force

$$
F_{t}=\frac{F_{T}}{n}=\frac{N}{n} \sin (\alpha),
$$

and an axial force

$$
F_{a}=\frac{F_{A}}{n}=\frac{N}{n} \cos (\alpha) .
$$

Thus the bending stiffness of a single beam is

$$
K_{\mathrm{b}}=\frac{12 E I}{L^{3}}+\frac{6 F_{a}}{5 L} .
$$

The stiffness in traction of a single beam is equal to

$$
K_{\text {tra }}=\frac{w t E}{L} .
$$

so the elongation due to traction is here equal to

$$
u_{\text {tra }}=\frac{F_{a}}{K_{\text {tra }}},
$$

Finally, the torsional stiffness of the beam is

$$
K_{\mathrm{tor}}=\frac{4 I G}{L},
$$

with $G=\frac{E}{2(1+\mu)}, E$ the Young's modulus, and $\mu$ the Poisson's ratio. The beams are submitted to a torsion angle $\beta$ equal to

$$
\beta=\arccos \left(\mathbf{v} \cdot \mathbf{v}^{\prime}\right),
$$

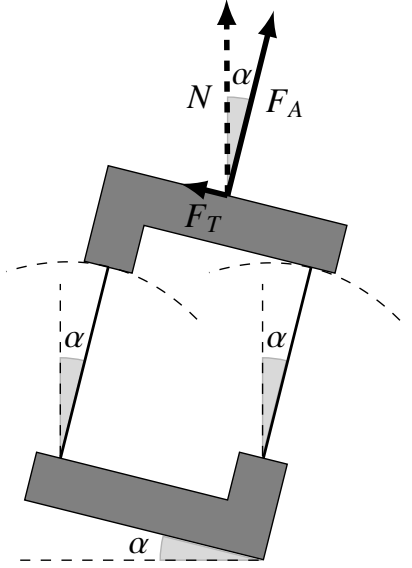

Figure 8: Determination of the bending and traction load applied on the CWM.

with

$$
\mathbf{v}=\operatorname{ROT}_{x}(\alpha) \times \mathbf{y},
$$

and

$$
\mathbf{v}^{\prime}=\operatorname{ROT}_{z}(\theta) \times \operatorname{ROT}_{x}(\alpha) \times \mathbf{y},
$$

where $\operatorname{ROT}_{x}(\alpha)$ corresponds to the rotation matrix for an angle $\alpha$ around $\mathbf{x}$ and $\operatorname{ROT}_{z}(\theta)$ corresponds to the rotation matrix for an angle $\theta$ around $\mathbf{z}$. The expression of $\beta$ is therefore:

$$
\beta=\arccos \left(\sin \left(\alpha^{*}\right) \sin (\alpha)+\cos \left(\alpha^{*}\right) \cos (\alpha) \cos (\theta)\right),
$$

with $\alpha^{*}$ conjugated of $\alpha$.

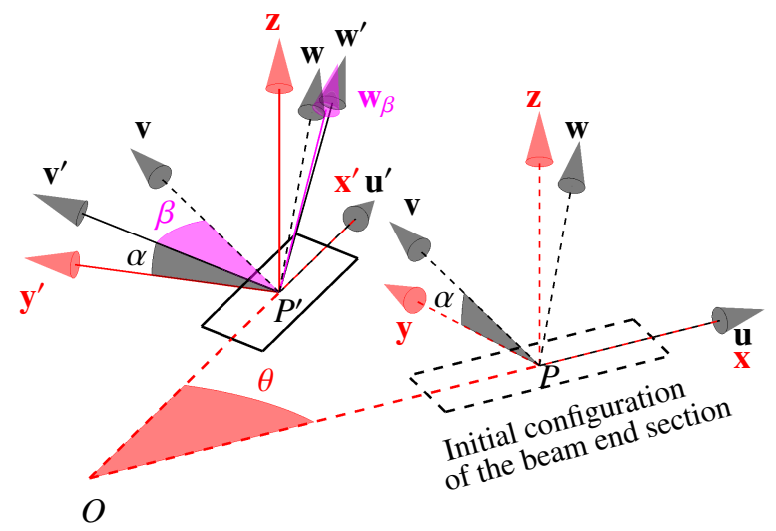

Figure 9: Estimation of the torsion angle $\beta$ for given angles $\theta$ and $\alpha$.

Using the principle of energy conservation, it is then possible to determine the motion of the CWM. The strain energy $E_{\mathrm{D}}$ for producing a displacement $f$ of a 
single blade is

$$
E_{\mathrm{D}}=E_{\mathrm{b}}+E_{\mathrm{tor}}+E_{\mathrm{tra}}=\frac{1}{2}\left(K_{b} f^{2}+K_{\mathrm{tor}} \beta^{2}+K_{\mathrm{tra}} u_{\mathrm{tra}}^{2}\right) .
$$

This energy can also be expressed as the product between $F_{t}$ and the displacement $f$ :

$$
E_{\mathrm{D}}=\frac{1}{2} F_{t} \times f,
$$

which leads to

$$
F_{t}=\frac{K_{b} f^{2}+K_{\mathrm{tor}} \beta^{2}+K_{\mathrm{tra}} u_{\mathrm{tra}}^{2}}{f} .
$$

One has then the relationship between the axial load $N$ on the CWM and the tangential deflection $f$ the beams:

$$
\frac{N}{n} \sin (\alpha)=\frac{K_{b}\left(F_{a}\right) f^{2}+K_{\text {tor }}(\beta(\theta))^{2}+K_{\text {tra }} F_{a}}{f} .
$$

where $\theta$ is function of $f$ see Eq. (6) and $F_{a}=\frac{N}{n} \cos (\alpha)$. For a given value of $N$, the tangential deflection $f$ can then be determined numerically. The CWM axial displacement $u$ and rotation $\theta$ can then be obtained using Eq. (7) and (6).

\subsubsection{Stress determination}

The stress in the beams of the equivalent parallelspring stage during bending is

$$
\sigma_{b}=3|f|\left(\frac{E t}{L^{2}}-F_{a} \frac{\pi^{2}-12}{w t^{2} \pi^{2}}\right) .
$$

The stress due to torsion is

$$
\sigma_{\text {tor }}=\frac{|\beta| t G \sqrt{3}}{L}
$$

The stress due to traction is

$$
\sigma_{\text {tra }}=\frac{\left|F_{a}\right|}{w t}
$$

For the design, we build a stress estimator by directly summing these contributions

$$
\sigma_{\text {max }}=\sigma_{b}+\sigma_{\text {tor }}+\sigma_{\text {tra }}
$$

This might be an overestimation as the location of maximum stresses is not necessarily the same for bending, traction and torsion in case of a curved beam. In addition, these expressions do not integrate the possible presence of stress concentrations, and the material sensitivity to longitudinal stress or shear stress is not taken into account by computing an equivalent stress.

\section{Additive manufacturing strategy}

The elaboration of an additive manufacturing strategy for CWM includes the selection of adequate AM process parameters and the identification of design guidelines so CWM can be produced with SLM. An iterative experimental approach was adopted to elaborate this strategy. The parameters of importance for manufacturing appear to be i) the 3D-design in the axial direction of the CWM, ii) the placement and orientation of the structure on the building platform during SLM manufacturing, iii) the realization of the support structures to the building platform and iv) the process parameters like laser energy and scanning trajectory. Material, methods and the derived design guidelines are introduced below.

\subsection{Materials and methods}

For the additive manufacturing, the 3D CAD models were designed with SolidWorks (2017 SP3, Dassault Systemes France). The support structures were created with Magics (V21, Materialise) using adjusted line support. The manufacturing of the parts was done using a SLM 250 HL system by SLM Solutions $\mathrm{GmbH}$ (Lübeck, Germany) with a building platform of $250 \times 250 \mathrm{~mm}^{2}$, with integrated powder reconditioning and sieving unit. A layer height of $50 \mu \mathrm{m}$ was used to print a pure titanium grade 2 powder (SLM-Solutions $\mathrm{GmbH}$ ) with a $\mathrm{d}_{50}$ of $41 \pm 2 \mu \mathrm{m}$ (Particle size analysis with Helos/KF + RODOS + VIBRI particle size distribution analysis set up by SympaTec $\mathrm{GmbH}$, ClausthalZellerfeld, Germany). The printing parameters were $100 \mathrm{~W}$ nominal laser power for the outer contour at a scanning speed of $550 \mathrm{~mm} / \mathrm{s}$ and $175 \mathrm{~W}$ for the inner contour with a scanning speed of $833 \mathrm{~mm} / \mathrm{s}$. The 4$\mathrm{mm}$ hatching was done in a checker-board pattern using $100 \mathrm{~W}$ laser power and $550 \mathrm{~mm} / \mathrm{s}$ scanning speed [21]. After the SLM process, the CWM were carefully detached manually from the building platform. The support structures were then broken off at predetermined breaking points and the contact points were machined with a lathe. Finally, the complete CWM was sandblasted (EKRA $\mathrm{Al}_{2} \mathrm{O}_{3}, 5$ bar) to remove residual powder on the surface and compact the surface.

\subsection{Design modifications for SLM}

SLM manufacturing of CWM requires the printing of very delicate structures. Beam thickness needs to be minimal in order to increase possible motions. As represented in figure 1 the typical beam thickness is less than $0.5 \mathrm{~mm}$, with a large aspect ratio $(1: \geq 50)$, connecting bulkier sections like the top and bottom volumes. Therefore special care is needed to support the CWM 
beams. These beams with long aspect ratios must have enough mechanical strength to withstand shear forces applied by the re-coater for powder spreading during the SLM-process. Therefore, support structures are holding them in place and also dissipate the induced heat during the laser process. A first adaptation to the design schematically represented in figure 2 is the radial displacement of the beams of module 2 with respect to module 1. It is then possible to connect them to the building platform with separate support structures that do not interfere with the struts or with each other (Fig. 10 and 11).

However, it is paramount that the support structures are not too strong or stand on other struts in order to avoid damaging the beams upon removal [22]. Furthermore, the quick cross sectional changes in transition from thin struts to the bulky rings is challenging due to thermally-induced stresses. Therefore, in a second adaption the top surface of the component is constructed in a lightweight design and a smoothed cross section change (Fig. 10 in order to minimize its volume and the laser energy introduced into the layer thereby. All upper, rotating struts are attached to the perimeter of the end surface. This allows the top surface to be supported by central structures that do not interfere with the beams of the two CWM, see the blue pillars in figure 11 . The rather solid support structures of the end plate can be removed easily by twisting, however, a roughness remains on the inside of the top surface. Yet, the overall design allows this surface to be machined with a lathe in order to turn away the support remnants without damaging the beams, thus creating a smooth contact surface for the pneumatic chamber (Fig 12).

The CWM behavior is dependent on the mechanical properties of the struts. The size and geometry of the beams need therefore be accurately controlled. For the bottom CWM (module 2 on figure 2), the inclination angle of the beams is small. It is then difficult to control the thickness of the beams because of the unfavorable overhang. As the heat conductivity of the powder bed located below the CWM is much lower compared to the CWM structure itself, the laser energy is dissipated insufficiently through the thin beams. This leads to a slower cooling rate and therefore increased temperature and volume of the melt pool. It was observed that this effect leads to an unwanted increase of the beams thickness. In early prototypes, the initial deviations reached $250-400 \mu \mathrm{m}$ ( $>50 \%$ of an aimed thickness of $500 \mu \mathrm{m})$. It was then identified that CAD model have to be scaled down accordingly. With this pre-process modification, deviations are lowered to only 50-150 $\mu \mathrm{m}$ (20\%). Nevertheless, the beams have a very coarse bottom surface

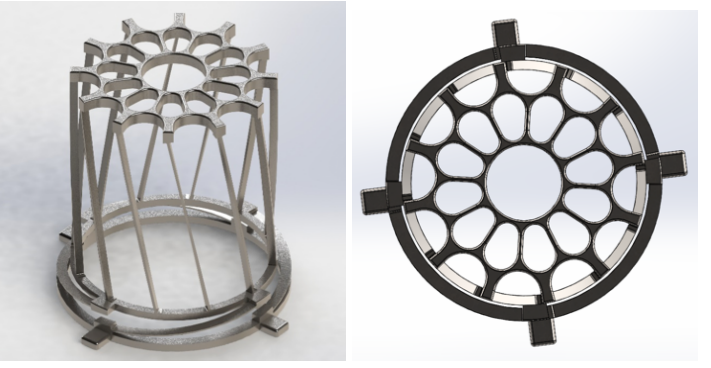

Figure 10: CAD rendering of the adapted, 3D-printable CWM in perspective (left) and top view of the light-weight end plate (right).

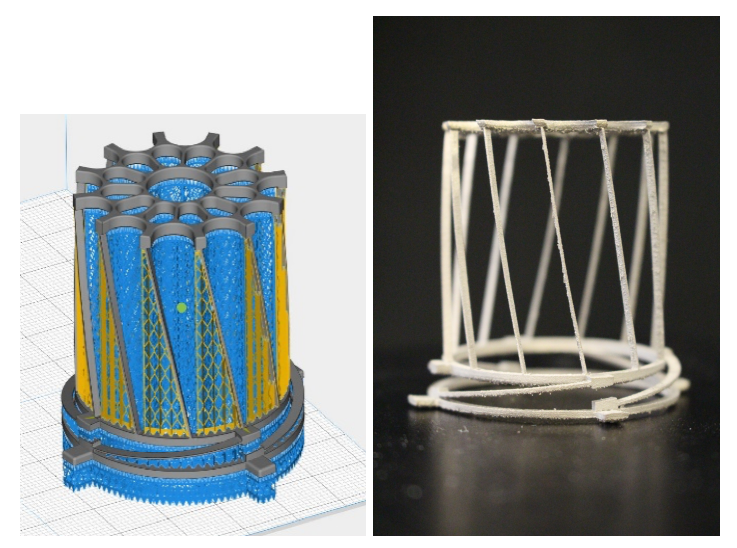

Figure 11: (left) CWM structure with support design ready for the SLM building process: the blue pillars are support structures that carry the horizontal end surface, the translation, lower beams and the lower ring, yellow highlighted supports bear the rotating, upper beams. (right) photo of a finished CWM structure after all support structures are removed, however before sandblasting.

as it is common for the process.

\section{Application}

\subsection{General architecture of the component}

From figure 6, one can see that the rotation $\alpha$ obtained with a CWM is controlled by the inclination angle $\alpha$. For small displacements around the origin, as encountered with compliant structures, the rotation is maximal when $\alpha=90^{\circ}$ and is close to zero when $\alpha \approx 0^{\circ}$.

In addition, the inclination angle $\alpha$ also impacts the axial stiffness of CWM. For same geometry of CWM beams, following Eq. 24 and Eq. 25, a CWM with horizontal beams $\left(\alpha=90^{\circ}\right)$ has the lowest stiffness possible, and on a contrary a CWM with vertical beams $\left(\alpha=0^{\circ}\right)$ has the highest stiffness.

The two modules of the actuator introduced in section 2 can then be chosen based on these observations. The module 1 depicted in figure 2 is a CWM with quasihorizontal beams. It is a low-stiffness CWM producing 


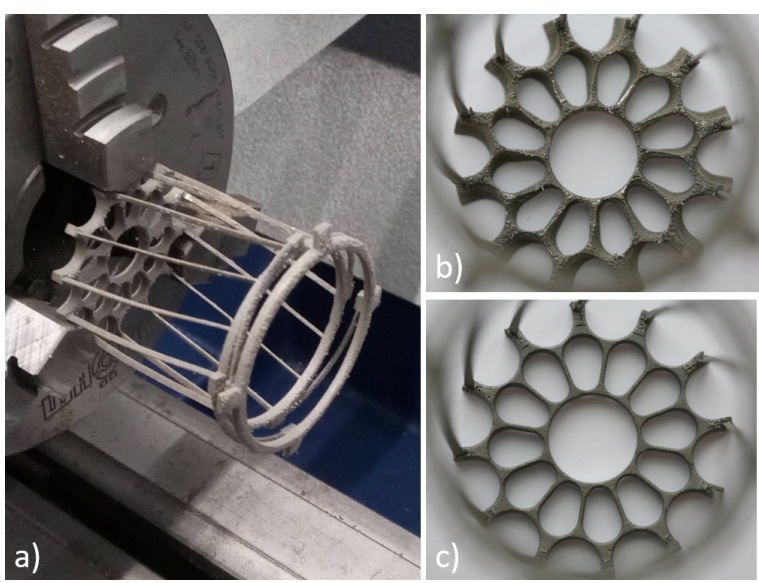

Figure 12: a) CWM in lathe for machining of the contact surface. b) Contact surface before machining. c) contact surface machined and sandblasted.

mainly a translation output motion when it is submitted to a vertical force generated by a pneumatic chamber. Conversely, the module 2 is a CWM with quasi-vertical blades. It is a CWM with high axial stiffness producing mainly a rotation output motion. The resulting difference of axial stiffness between the two modules makes it possible to produce the translation and the rotation by changing the amplitude of the axial force generated with the pneumatic chamber.

Each CWM cannot produce a pure translation or rotation. In order to ensure translation, we add as a design objective in the design of the component that the parasitic rotation of the module 1 has to be compensated by the rotation produced by the module 2 as it is mounted on top of module 1 and it can have opposite pitch. This means we set the total rotation of the component at pressure $P_{\mathrm{t}}$, for which the module 1 is reaching the mechanical stopper (Fig. 2), to be equal to zero.

The design of the pneumatic chamber is quite decoupled from the the design of CWM in the component. The chamber needs to be of annular shape to have on one hand the needle going through the center, and on the other hand the CWM placed around the chamber. A simple design based on a succession of rigid and soft rings is chosen, as depicted in figure 14. For the proof of concept, the stiffness of rings is modified by choosing materials of different stiffnesses. Multi-material additive manufacturing is considered (Polyjet technology, Stratasys) with use of VeroWhite Plus and TangoBlack Plus materials.

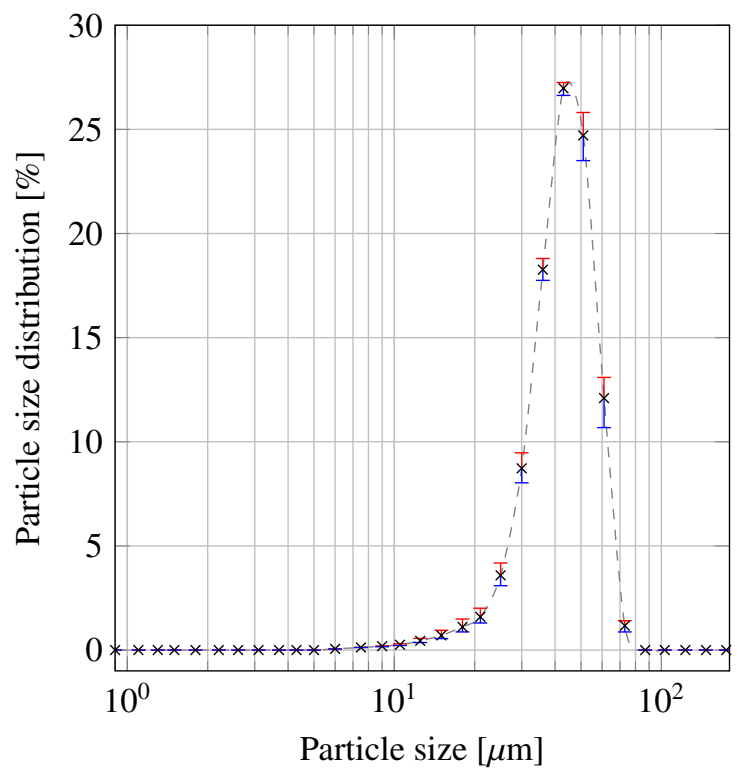

Figure 13: Particle size distribution of the used Ti powder grade 2.

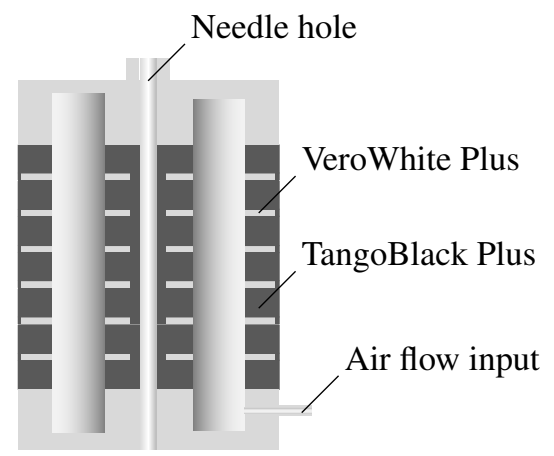

Figure 14: Design of the pneumatic chamber.

\subsection{Design of the component}

The design objectives were introduced in section 2 : the desired translation and rotation should reach $2 \mathrm{~mm}$ and $5^{\circ}$ for an input pressure of 3 bars.

These objectives have to be fulfilled with a compact device given the integration in a medical environment [17]. The maximum radius and height are set to $22 \mathrm{~mm}$ and $45 \mathrm{~mm}$. The production with SLM constraints the thickness and width of beams in the CWM to be larger than $0.5 \mathrm{~mm}$.

The synthesis of the component was achieved iteratively, using these design objectives and constraints. The CWM design parameters of the two modules are indicated in tables 1 and 2 .

The relationship between the axial displacement and 
Table 1: CWM parameters for the module 1.

\begin{tabular}{|c|c|c|c|c|c|c|c|c|c|}
\hline$n$ & $R[\mathrm{~mm}]$ & $L[\mathrm{~mm}]$ & $\alpha\left[^{\circ}\right]$ & $H[\mathrm{~mm}]$ & $p\left[\mathrm{~mm} /{ }^{\circ}\right]$ & $w[\mathrm{~mm}]$ & $t[\mathrm{~mm}]$ & $E[\mathrm{MPa}]$ & $v$ \\
\hline 4 & 21 & 27 & 83 & 3.3 & 0.045 & 2 & 0.5 & 106900 & 0.3 \\
\hline
\end{tabular}

Table 2: CWM parameters for the module 2 .

\begin{tabular}{|c|c|c|c|c|c|c|c|c|c|}
\hline$n$ & $R[\mathrm{~mm}]$ & $L[\mathrm{~mm}]$ & $\alpha\left[^{\circ}\right]$ & $H[\mathrm{~mm}]$ & $p\left[\mathrm{~mm} /{ }^{\circ}\right]$ & $w[\mathrm{~mm}]$ & $t[\mathrm{~mm}]$ & $E[\mathrm{MPa}]$ & $v$ \\
\hline 12 & 18.7 & 40 & -11 & 39.3 & -1.7 & 1.5 & 0.5 & 106900 & 0.3 \\
\hline
\end{tabular}
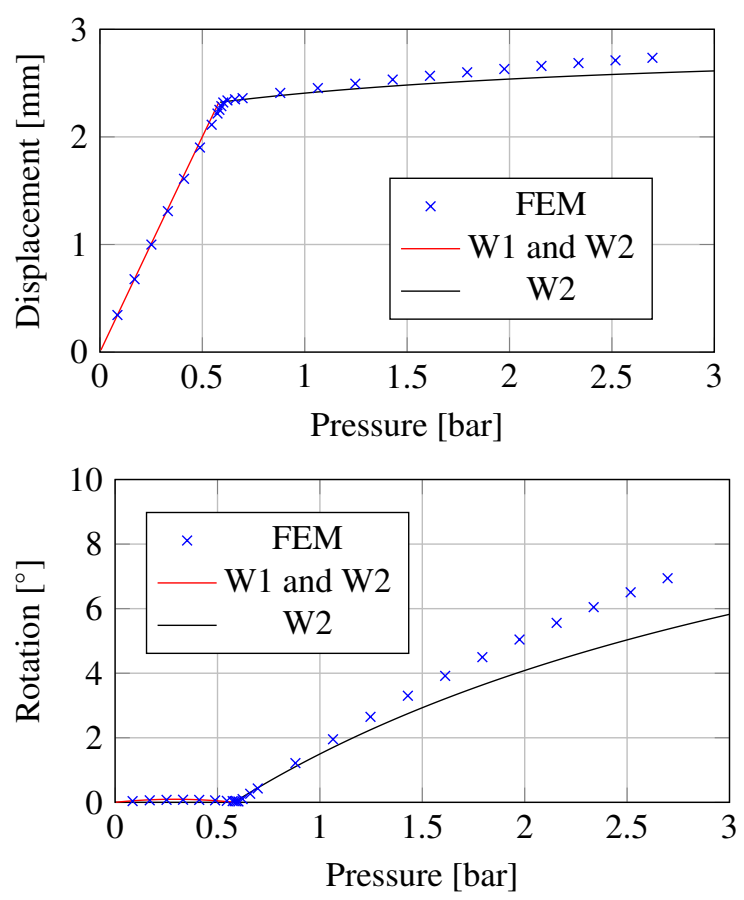

Figure 15: Displacement and rotation as function of the pressure with the developed models and the FEM. Blue crosses correspond to the FEM results. The red line corresponds to the motion of modules 1 and 2 , the black line is due to the motion of the module 1 .

the output rotation as obtained thanks to the developed CWM models is represented in figure 18. The results obtained with a finite element model (FEM) of the component behavior, using Creo/Simulate (PTC, USA), are also indicated on the figure. The displacement-pressure and rotation-pressure curves are provided on figure 15 .

Animation of the component displacement under application of the pneumatic pressure is provided in the attached video. In figures 16 and figure 17, the configuration under the application of 2.7-bar and 0.57 bar pressure are represented with the Von Mises stress in the structure. For this situation, the FEM value is $764 \mathrm{MPa}$, the maximum yield stress of the material being equal to $750 \mathrm{MPa}$. One can notice from figure 18 that the de- veloped models describe with a very good accuracy the kinematic behavior of the component. At $p_{t}=0.57 \mathrm{bar}$ (Fig. 15), which is the maximum pressure for which the two CWM contribute to the total translation, the relative errors in the estimation of the displacement is equal to $1 \%$. The rotation is well compensated with $0.05^{\circ}$ for the analytical output axial rotation estimation versus $0.03^{\circ}$ in the finite element simulations. When the module 1 reaches its maximum translation, for a pressure of 2.7 bars, the relative errors in the estimation of the displacement and rotation are respectively equal to $4 \%$ and $14 \%$. The latter discrepancy is due to the parabolic relation between rotation and translation. In terms of kinematics, the prediction is very well estimated as for the same output rotation of $7^{\circ}$, the error in the displacement is less than $3 \%$.

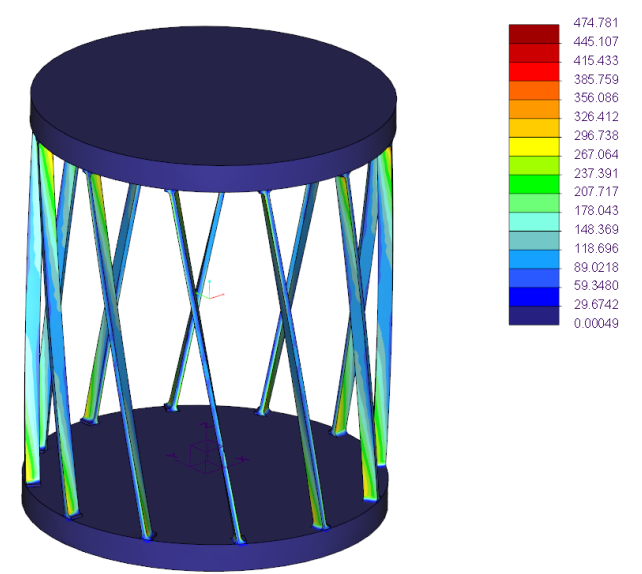

Figure 16: Von Mises stress distribution in the CWM1 at 2.7 bar.

In addition, the design parameters allow us to satisfy the requirements with an axial displacement using the module 1 equal to $2.3 \mathrm{~mm}$. When the mechanical stopper comes into contact with the module 1 , the rotation is compensated $\left(\theta=0.03^{\circ}\right.$ according to the FEM). The total rotation for a pressure of 3 bars is equal to $6^{\circ}$ according to the analytical model, which is above the objective of $5^{\circ}$. 

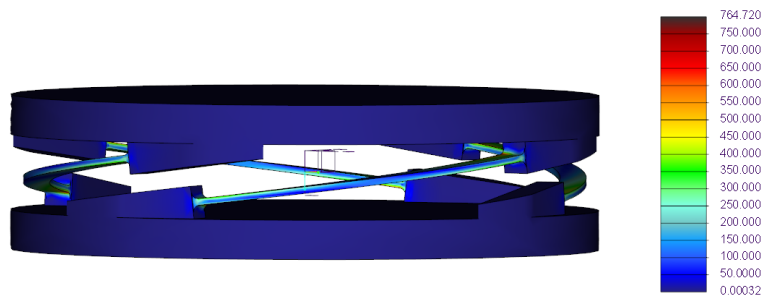

Figure 17: Von Mises stress distribution in the CWM2 at $p_{t}=$ 0.57 bar.

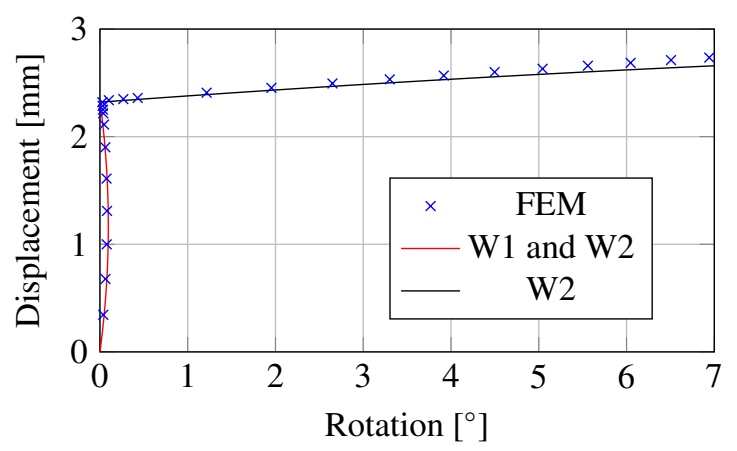

Figure 18: Displacement-rotation relationship with the proposed component. Blue crosses correspond to the FEM results. The red line corresponds to the motion of modules 1 and 2, the black line is due to the motion of the module 1 .

\subsection{Experimental evaluation}

The experimental setup is depicted in figure 20. The prototype is rigidly mounted to the base. The axial output motion $u$ is measured with a laser sensor (OptoNCDT 2300 series, Micro-Epsilon) with 8 micron resolution and recorded with a computer. The output axial rotation $\theta$ is measured visually with a protractor with a resolution of $0.5^{\circ}$. The input pressure is controlled with a circuit composed of a proportional valve (VPPM series, Festo) for the pressure regulation and a fast 3/2way solenoid valve (MHE2 series, Festo) to trigger the output. The air pressure delivered by this stage is measured with a pressure sensor (SPTW series, Festo). A custom software running under a real-time operating system (Linux Xenomai) is then used to adjust the pressure level.

The measurements are performed as follows. Without input air pressure, the initial axial position and rotation are recorded and defined as initial offsets. Then, the pressure is increased by increments of 0.1 bar and recorded with the axial position measurements in order to determine the axial motion $u$. The angular output position is recorded manually with the corresponding pressure to extract the axial rotation $\theta$.
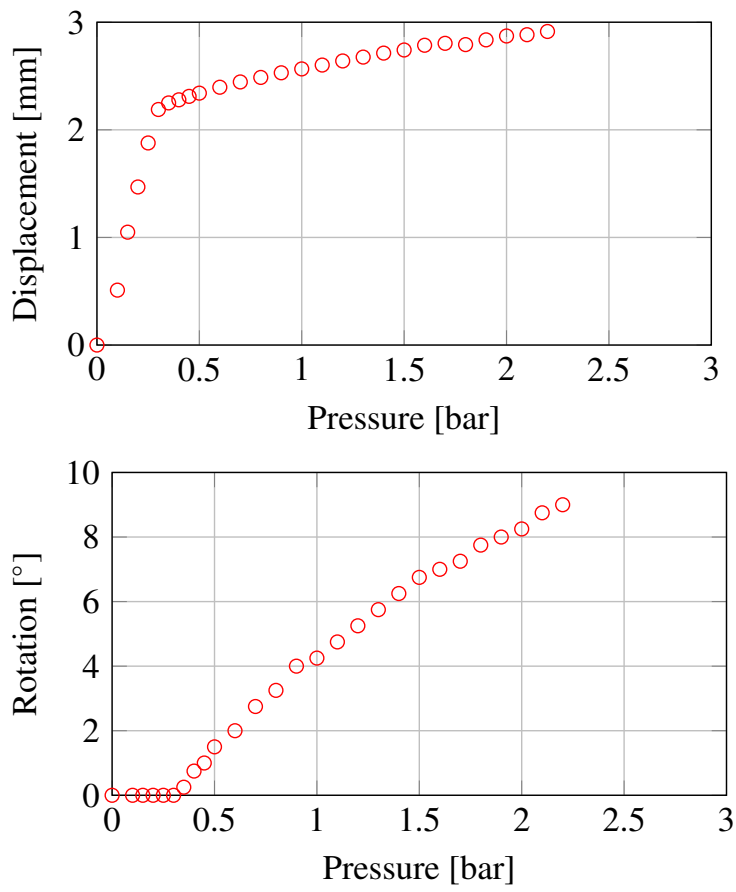

Figure 19: Experimental measurements of the axial output displacement and rotation

The results of the axial motion and rotation with respect to the input pressure are indicated by red circles in figure 19. The evolution of displacement and rotation is in accordance with the model predictions. The axial stiffness of the prototype appears however lower than expected, so the total rotation of $9^{\circ}$ is for instance higher than expected. The difficulty to control the thickness of flexible beams as observed in the previous section (up to $20 \%$ decrease on the thickness) can largely contribute to this stiffness decrease. In fact, if we consider the particular case were all blades are $20 \%$ thinner, the actuation pressure to reach a $9^{\circ}$ rotation would be according to the model equal to 2.7 bars instead of 5 bars. The stiffness is then almost divided by two.

The kinematic behavior linking the axial rotation and the displacement of the device is given in figure 21. One can see the interesting accordance with the proposed models and the FEM prediction. Thanks to the design of the component, it is possible to get sufficient translation and rotation for the application context. The translation is obtained for a given range of pressure, and a pressure increase allows to control the rotation. The two working modes are obtained with a single input pressure. 


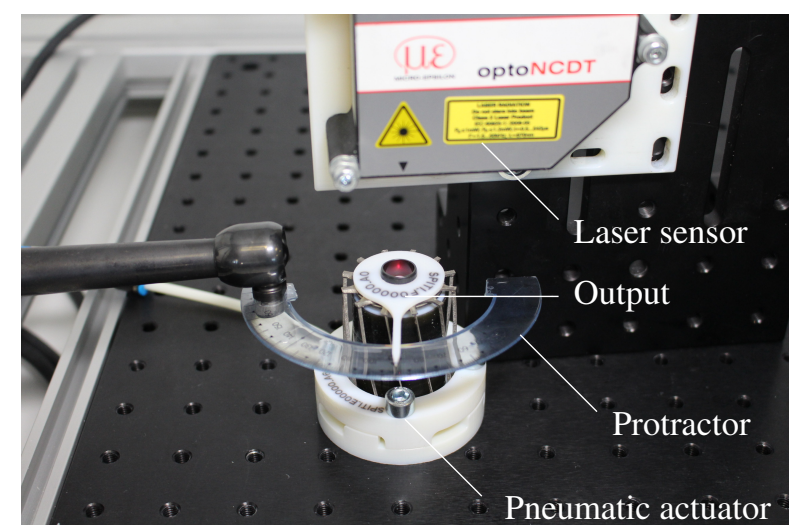

Figure 20: Experimental set-up.

\section{Conclusions}

In this article, we investigated the modeling and production of CWM. These compliant structures are of interest for the specific motions they provide, which is illustrated by the development of an actuator for biomedical applications. Kinematic and static models have been developed so the designer can adjust the geometry of CWM for given requirements. The accuracy of the proposed models appears satisfactory when comparing the model prediction to FEM results and the experimental characterization achieved for the biomedical application. Two separate models have been introduced, each one corresponding to a type of geometry of possible interest. One perspective is to merge these models and develop a fully generic description of the CWM behavior. Another perspective concerns the combination of CWM or the addition of other compliant structures to guide the motion to get for instance a pure rotation as in [5], or to combine sets of flexible beams to suppress infinitesimal motions as envisioned in [23].

Thanks to careful planning of the geometry and adjustments to the strengths and limitations of additive manufacturing, the CWM structure could be manufactured using SLM. The positioning of the support structures for mechanical and thermal connection to the construction platform was particularly important. The laser and process parameters were carefully worked out so that the planned structure could be manufactured in the specified geometric and thus mechanical specifications. Various other approaches could be considered in the future to improve the SLM-fabrication process further. There are equipment based modifications, like e.g. finer powders and lasers. Other approaches could be investigated like automated design optimization, optimal orientation of the part on the printing platform or special
Axial displacement-rotation

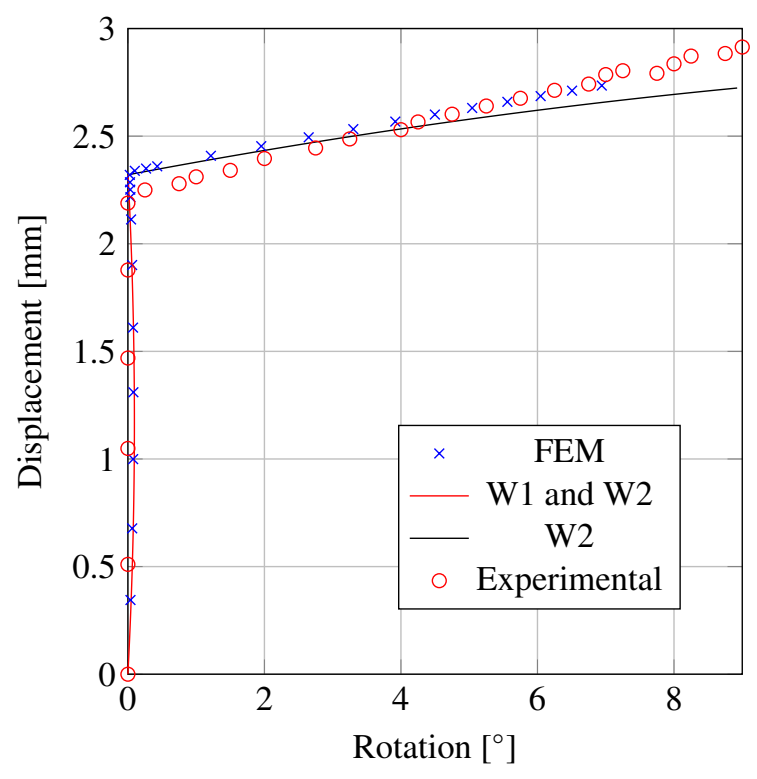

Figure 21: Comparison of the experimental actuator kinematics with the analytical model and the finite element simulations.

support designs [22] as they were used in part in this project. More advanced process approaches like preprocess calculation of local process parameters [15] or geometry [24] are commercially available. Approaches using in situ online process control [25] are still a topic of research. All these approaches have their distinct advantages but rarely one approach solves all problems, so most likely combinations will be used in the future.

One field of application of the developed additivelymanufactured CWM is in medical and biomedical devices. The currently rapid improvement of manufacturing processes will make the CWM easier to manufacture and widen the potential use cases. Therefore one perspective is also to push forward the development of CWM-based components for industrial or medical applications in the near future.

\section{Acknowledgements}

This work was supported by the INTERREG Upper Rhine program from the ERDF (European Regional Development Fund), SPIRITS project, and also by Investissements d'Avenir program (Labex CAMI \& Equipex ROBOTEX) as well as by the cantons Baselstadt, Basellandschaft, Aargau and the Swiss Confederation. 


\section{References}

[1] J.-P. Merlet, Parallel robots, Vol. 74, Springer Science \& Business Media, 2001.

[2] Y. Qin, J. Dai, Forward displacement analysis of two foldable 3us parallel mechanisms, Advances in Reconfigurable Mechanisms and Robots I (2012) 805-814doi:10.1007/ 978-1-4471-4141-9_72

[3] L. Rubbert, I. Charpentier, S. Henein, P. Renaud, Higher-order continuation method for the rigid-body kinematic design of compliant mechanisms. Precision Engineering 50 (2017) 455466. doi:https://doi.org/10.1016/j.precisioneng. 2017.06 .021

URL https://www.sciencedirect.com/science/ article/pii/S0141635917300983

[4] T. Gayral, L. Rubbert, P. Renaud, Modeling and identification for the design of a rotary soft actuator based on wren mechanism, in: Proceedings of the IEEE/RSJ International Conference on Intelligent Robots and Systems, 2019.

[5] R. Wang, X. Zhou, Z. Zhu, Q. Liu, Compliant linear-rotation motion transduction element based on novel spatial helical flexure hinge, Mechanism and Machine Theory 92. doi:10.1016/ j.mechmachtheory.2015.06.005

[6] R. Vertechy, V. Parenti Castelli, Static and stiffness analyses of a class of over-constrained parallel manipulators with legs of type us and ups, 2007, pp. $561-567$. doi:10.1109/ROBOT. 2007. 363046

[7] G. Kiper, E. Söylemez, Modified wren platforms, 2011.

[8] K. Wohlhart, Kinematotropic Linkages, Springer Netherlands, Dordrecht, 1996, pp. 359-368. doi:10.1007/ 978-94-009-1718-7_36

[9] K. Zhang, J. S. Dai, Reconfiguration analysis of wren platform and its kinematic variants based on reciprocal screw systems, in: Proceedings of the 14th IFToMM World Congress, 2015, pp. 1070-1076.

[10] A. De Greef, P. Lambert, A. Delchambre, Towards flexible medical instruments: Review of flexible fluidic actuators, Precision Engineering 33 (4) (2009) 311-321.

[11] A. A. Calderón, J. C. Ugalde, J. C. Zagal, N. O. PérezArancibia, Design, fabrication and control of a multi-materialmulti-actuator soft robot inspired by burrowing worms, 2016, pp. 31-38.

[12] D. Comber, J. Slightam, V. Gervasi, J. Neimat, E. Barth, Design, additive manufacture, and control of a pneumatic, mrcompatible needle driver, IEEE Transactions on Robotics 32. doi:10.1109/TRO.2015.2504981

[13] A. Pfeil, L. Barbé, B. Wach, A. Bruyas, F. Geiskopf, M. Nierenberger, P. Renaud, A 3D-printed needle driver based on auxetic structure and inchworm kinematics, in: ASME 2018 Int. Design Eng. Tech. Conf., 2018.

[14] C. Andersen, S. Magleby, L. Howell, Principles and preliminary concepts for compliant mechanically reactive armor, 2009, pp. $370-376$.

[15] N. Matter, Pre-process calculation to optimize laser parameter in selective laser melting (Jun. 2018).

URL https://www.ecmconferences.org/abstracts/ 2018/Collection2/c2.html

[16] C. Qiu, S. Yue, N. J. E. Adkins, M. Ward, H. Hassanin, P. D. Lee, P. J. Withers, M. M. Attallah, Influence of processing conditions on strut structure and compressive properties of cellular lattice structures fabricated by selective laser melting Materials Science and Engineering: A 628 (2015) 188 - 197. doi:https://doi.org/10.1016/j.msea.2015.01.031

URL http://www.sciencedirect.com/science/ article/pii/S0921509315000453
[17] A. Pfeil, L. Barbé, B. Wach, C. R.L., A. Gangi, P. Renaud, Observations and experiments for the definition of a new robotic device dedicated to ct, cbct and mri-guided percutaneous procedures, in: IEEE Engineering in Medicine and Biology Conference, 2018, pp. 1708-1712.

[18] N. J. van de Berg, D. J. van Gerwen, J. Dankelman, J. J. van den Dobbelsteen, Design Choices in Needle Steering-A Review, IEEE/ASME Transactions on Mechatronics 20 (5) (2015) 2172 2183.

[19] B. Maurin, B. Barbé L., Bayle, P. Zanne, J. Gangloff, M. de Mathelin, A. Gangi, L. Soler, A. Forgione, In vivo study of forces during needle insertions, in: Proceedings of the Medical Robotics, Navigation and Visualisation Scientific Workshop, 2004, pp. 415-422.

[20] L. Rubbert, R. Bitterli, N. Ferrier, S. Fifanski, I. Vardi, $\mathrm{S}$. Henein, Isotropic springs based on parallel flexure stages, Precision Engineering 43 (2016) 132 - 145. doi:https: //doi.org/10.1016/j.precisioneng.2015.07.003

[21] M. de Wild, R. Schumacher, K. Mayer, E. Schkommodau, D. Thoma, M. Bredell, A. Kruse Gujer, K. W. Grätz, F. E. Weber, Bone Regeneration by the Osteoconductivity of Porous Titanium Implants Manufactured by Selective Laser Melting: A Histological and Micro Computed Tomography Study in the Rabbit Tissue Engineering Part A 19 (23-24) (2013) 26452654, _eprint: https://doi.org/10.1089/ten.tea.2012.0753. doi: 10.1089/ten.tea.2012.0753 URL https : //doi.org/10.1089/ten.tea.2012.0753

[22] T. Maconachie, M. Leary, B. Lozanovski, X. Zhang, M. Qian, O. Faruque, M. Brandt, SLM lattice structures: Properties, performance, applications and challenges Materials \& Design 183 (2019) 108137. doi:https: //doi.org/10.1016/j.matdes.2019.108137 URL http://www.sciencedirect.com/science/ article/pii/S0264127519305751

[23] J. Hopkins, J. Vericella, C. Harvey, Modeling and generating parallel flexure elements, Precision Engineering 38. doi:10. 1016/j.precisioneng.2014.02.001

[24] Simufact Additive (May 2020). URL https : //www . simufact . com

[25] Amphyon(May 2020). URL https : //additive.works 


\section{Appendix}

Table 3: List of parameters and notations for the CWM modeling.

\begin{tabular}{|c|c|}
\hline Parameter & Description \\
\hline \multicolumn{2}{|r|}{ Geometry } \\
\hline$n$ & Number of beams \\
\hline$H[\mathrm{~m}]$ & Height of the CWM \\
\hline$R[\mathrm{~m}]$ & Distance of beam neutral fiber to the axis $Z$ \\
\hline$p[\mathrm{~m} / \mathrm{rad}]$ & Pitch \\
\hline$t[\mathrm{~m}]$ & Beam thickness \\
\hline$w[\mathrm{~m}]$ & Beam width \\
\hline$L[\mathrm{~m}]$ & Length of the beam neutral fiber \\
\hline$\alpha[\mathrm{rad}]$ & Vertical inclination angle of the beam \\
\hline \multicolumn{2}{|r|}{ Kinematics } \\
\hline$u[\mathrm{~m}]$ & CWM axial motion \\
\hline$\theta[\mathrm{rad}]$ & CWM axial rotation \\
\hline$f[\mathrm{~m}]$ & Parallel spring stage lateral displacement \\
\hline$\lambda[\mathrm{m}]$ & Parallel spring stage vertical displacement \\
\hline \multicolumn{2}{|r|}{ Material } \\
\hline$E[\mathrm{~Pa}]$ & Young's modulus \\
\hline$\mu$ & Poisson's ratio \\
\hline \multicolumn{2}{|r|}{ Beam loading, deformations and stresses } \\
\hline$I\left[\mathrm{~m}^{4}\right]$ & Beam area moment of inertia \\
\hline$N[\mathrm{~N}]$ & Axial load on the CWM \\
\hline$K_{c}\left[\mathrm{~N} \mathrm{~m}^{-1}\right]$ & CWM axial stiffness \\
\hline$K_{b}\left[\mathrm{~N} \mathrm{~m}^{-1}\right]$ & Bending stiffness of one beam \\
\hline$K_{\text {tra }}\left[\mathrm{N} \mathrm{m}^{-1}\right]$ & Traction stiffness of one beam \\
\hline$K_{\text {tor }}\left[\mathrm{N} \mathrm{m}^{-1}\right]$ & Torsional stiffness of one beam \\
\hline$F_{t}[\mathrm{~N}]$ & Tangential force on the parallel spring stage \\
\hline$F_{a}[\mathrm{~N}]$ & Axial force on the the parallel spring stage \\
\hline$M_{f}[\mathrm{Nm}]$ & Bending moment along the beam neutral fiber \\
\hline$u_{\text {tra }}[\mathrm{m}]$ & Beam elongation due to traction \\
\hline$\beta[\mathrm{rad}]$ & Beam torsion angle \\
\hline$\sigma_{b}[\mathrm{~Pa}]$ & Stress due to bending \\
\hline$\sigma_{\text {tra }}[\mathrm{Pa}]$ & Stress due to traction \\
\hline$\sigma_{\text {tor }}[\mathrm{Pa}]$ & Stress due to torsion \\
\hline$E_{b}[\mathrm{~J}]$ & Deformation energy due to bending \\
\hline$E_{\text {tra }}[\mathrm{J}]$ & Deformation energy due to traction \\
\hline$E_{\text {tor }}[\mathrm{J}]$ & Deformation energy due to torsion \\
\hline
\end{tabular}

